\title{
Enhanced detergent extraction for analysis of membrane proteomes by two-dimensional gel electrophoresis Matthew A Churchward ${ }^{1}$, R Hussain Butt ${ }^{1}$, John C Lang1, Kimberly K Hsu ${ }^{1}$ and Jens R Coorssen*1,2,3
}

\begin{abstract}
Address: ${ }^{1}$ Dept. of Physiology and Biophysics, University of Calgary Faculty of Medicine, 3330 Hospital Drive NW, Calgary AB, T2N 4N1, CANADA, ${ }^{2}$ Dept. of Biochemistry and Molecular Biology, University of Calgary Faculty of Medicine, 3330 Hospital Drive NW, Calgary AB, T2N 4N1, CANADA and ${ }^{3}$ Hotchkiss Brain Institute, University of Calgary Faculty of Medicine, 3330 Hospital Drive NW, Calgary AB, T2N 4N1, CANADA

Email: Matthew A Churchward - mchurchw@ucalgary.ca; R Hussain Butt - rbutt@ucalgary.ca; John C Lang - johnclang@shaw.ca; Kimberly K Hsu - khsu3338@interchange.ubc.ca; Jens R Coorssen* - jcoorsse@ucalgary.ca

* Corresponding author
\end{abstract}

Published: 07 June 2005

Proteome Science 2005, 3:5 doi:10.1186/1477-5956-3-5

This article is available from: http://www.proteomesci.com/content/3/1/5

(C) 2005 Churchward et al; licensee BioMed Central Ltd.

This is an Open Access article distributed under the terms of the Creative Commons Attribution License (http://creativecommons.org/licenses/by/2.0), which permits unrestricted use, distribution, and reproduction in any medium, provided the original work is properly cited.
Received: 23 January 2005

Accepted: 07 June 2005

\begin{abstract}
Background: The analysis of hydrophobic membrane proteins by two-dimensional gel electrophoresis has long been hampered by the concept of inherent difficulty due to solubility issues. We have optimized extraction protocols by varying the detergent composition of the solubilization buffer with a variety of commercially available non-ionic and zwitterionic detergents and detergent-like phospholipids.
\end{abstract}

Results: After initial analyses by one-dimensional SDS-PAGE, quantitative two-dimensional analyses of human erythrocyte membranes, mouse liver membranes, and mouse brain membranes, extracted with buffers that included the zwitterionic detergent MEGA 10 (decanoyl-Nmethylglucamide) and the zwitterionic lipid LPC (I-lauroyl lysophosphatidylcholine), showed selective improvement over extraction with the common 2-DE detergent CHAPS (3 [(3cholamidopropyl)dimethylammonio]-I-propanesulfonate). Mixtures of the three detergents showed additive improvements in spot number, density, and resolution. Substantial improvements in the analysis of a brain membrane proteome were observed.

Conclusion: This study demonstrates that an optimized detergent mix, coupled with rigorous sample handling and electrophoretic protocols, enables simple and effective analysis of membrane proteomes using two-dimensional electrophoresis.

\section{Background}

Historically, the proteomic analysis of hydrophobic membrane proteins has been considered to be difficult within the bounds of conventional protocols for two-dimensional gel electrophoresis (2-DE). The nature of first dimension isoelectric focusing (IEF) requires that proteins be thoroughly solubilized as they are subjected to an elec- tric field in which they migrate to their isoelectric point, by definition the state of lowest possible net charge and thus lowest solubility in aqueous environments. In addition to being highly hydrophobic, many integral membrane proteins tend to be very large: human $\mathrm{Ca}^{2+}$ channels have 24 transmembrane helices and are typically $>200$ $\mathrm{kDa}[1]$, and tyrosine kinase receptors are frequently $>$ 
$100 \mathrm{kDa}[2,3]$. This leads to two major problems in the preparation of membrane protein samples for 2-DE. First, effectively extracting membrane proteins into a detergent that is IEF compatible. Second, maintaining protein solubility throughout loading onto IPG strips and the subsequent first dimension IEF separation. Although highly efficient membrane protein extractions are routinely carried out with a detergent such as SDS for one-dimensional PAGE, SDS is incompatible with IEF due to the charged head group. To overcome this, SDS solubilized samples often undergo solvent or acid precipitation to remove or reduce SDS and lipids. Despite these harsh treatments and even subsequent treatment of the precipitate with a strong base [4], delipidation by solvent extraction is often cited as enhancing protein recovery $[5,6]$ without discussion of the loss or modification of proteins during precipitation. For example, highly hydrophobic proteins (such as proteolipids) and proteins with particular post-translational modifications (such as palmitoylation) are capable of partitioning into the solvent phase [7-9], and TCA treatment can cause acid hydrolysis of proteins or alter post-translational modifications. Additionally, some early general problems with effectively separating hydrophobic proteins by 2-DE have led to widespread general disregard for the analysis of membrane proteins, particularly in the development of alternate proteomic approaches $[4,5,10,11]$.

Since membrane proteins comprise approximately $30 \%$ of human proteins[12], and may account for substantially more cellular functions, the focus on soluble proteins in so-called 'full' proteomic analyses is somewhat concerning. There is evidence that optimization of extraction conditions by alteration of buffers, chaotropes, and detergents is sufficient to reliably achieve high-resolution maps of membrane proteins [13-16]. To this end we have sought simple alternatives to optimize the detergent conditions used to extract proteins from native membranes by systematic analysis of the solubilization properties of a wide range of commercially available non-ionic and zwitterionic detergents and a range of natural and synthetic detergent-like lipids [17-19]. Using proven synthetic detergents, together with more native lipophilic agents, we find that combinations of these reagents generally improve the resolution of membrane proteomes analyzed by 2-DE, providing for select improvements in the yields of specific proteins. Optimization of conditions for particular samples remains a key to any successful analysis [2022].

\section{Results \& Discussion ID SDS-PAGE of RBC membrane}

Analysis of RBC extracts using 1D SDS-PAGE allowed for the rapid screening of a large number of extraction reagents (including glycerols, lipids, fatty acids, and isopre- noids), providing results that could be interpreted qualitatively based on the selective increase and decrease of protein banding patterns relative to control extractions with CHAPS or SDS (Fig. 1). For example, band III, a large protein with multiple transmembrane spanning domains [23] could be clearly distinguished in SDS extracts at an apparent MW of $\sim 110 \mathrm{kDa}$, compared to those made with CHAPS. Another band, at apparent MW of $28 \mathrm{kDa}$, was also observed in the SDS but not the CHAPS extract. Based on these simple criteria, detergents were selected that gave improved banding patterns over the CHAPS control extraction. An initial working series of effective detergents was thus identified for further testing (Table 1), and these were then used to extract RBC membrane samples for subsequent analysis by 2-DE. Notably LPC, the N-methylglucamide detergents MEGA 8, 9, and 10 and the sulfobetaine-based detergents ASB-14 and SB 3-10 showed improvements in the $1 \mathrm{D}$ banding pattern relative to the CHAPS control. A selection of natural source lipids were also tested, including lysophsphatidylglycerol (LPG), lysophosphatidylethanolamine (LPE), lysophosphatidylcholine (LPC, from egg), lysophosphatidylserine (LPS, from bovine brain), and cardiolipin (bovine heart). Although generally comparable, and of some selective use in extractions, most of these natural lipids proved to be of limited general usefulness as they are charged at neutral $\mathrm{pH}$, and thus inherently incompatible with IEF. This limitation does not obviate the potential application of these lipids as extraction agents for use with alternate protein separation paradigms.

\section{2-DE analysis of Red Blood Cell membrane}

Initially, red blood cell (RBC) membranes were extracted with a 2-DE buffer containing 4\% total CHAPS (our standard concentration) or $1-2 \%$ total detergent, due to the relatively lower solubility of most test detergents compared to the highly soluble CHAPS. Extraction of RBC membranes with synthetic LPC (lauroyl chain, Sigma) and the zwitterionic detergent MEGA 10 under these conditions resulted in areas of selective improvement in resulting 2-DE patterns relative to the control extracts in CHAPS (data not shown). 2-DE of samples extracted with SB 3-10 yielded similar protein maps to CHAPS, however this detergent was difficult to solubilize into high urea buffer, as previously reported [24,25]. However, contrary to earlier reports $[24,26]$, samples extracted with ASB-14 did not show improvement over either CHAPS or SB 3-10 (data not shown). In order to both appropriately account for more general effects of detergent concentration and take advantage of the high solubility and efficient solubilizing properties of CHAPS, both LPC and MEGA 10 were used to extract RBC membranes (Fig. 2), mouse brain membranes (Fig. 3), and mouse liver membranes (Fig. 5) as mixtures of 3\% CHAPS : $1 \%$ alternate detergent, for a total $4 \%$ detergent. 
Table I: Summary of detergents tested using systematic ID SDS-PAGE analysis. Overall extraction efficacy analyzed by ID SDSPAGE or 2-DE separation is expressed qualitatively relative to SDS extraction (for ID analysis) or CHAPS extraction (for 2-DE). + indicates compatibility but poor perfomance, ++ indicates similar or slightly worse than CHAPS extraction, +++ indicates performance equal to or better than CHAPS, - indicates incompatibility.

\begin{tabular}{|c|c|c|c|}
\hline Detergent & ID-PAGE & 2-DE & Comments \& Rationale \\
\hline SDS & +++ & - & IEF incompatible \\
\hline CHAPS & ++ & +++ & Poor extraction of hydrophobic and high molecular weight proteins \\
\hline trans, trans-farnesol & +++ & ++ & Natural isoprenoid \\
\hline MEGA-8a & +++ & ++ & Group of nonionic detergents commonly used for protein purification $[35,36]$ \\
\hline MEGA-9b & +++ & ++ & \\
\hline MEGA-I0c & +++ & +++ & \\
\hline amidosulfobetaine-14 (ASB-I4) & +++ & ++ & Sulfobetaine-based detergents reported to improve membrane protein extraction [24-26] \\
\hline Zwittergent ${ }^{\circledR} 3-10 / S B 3-10^{d}$ & +++ & +++ & \\
\hline LPC (synthetic, lauroyl chain)e & +++ & +++ & Zwitterionic lysophospholipid \\
\hline LPC (egg, mixed chain)e & ++ & ++ & Zwitterionic lysophospholipid \\
\hline LPS (bovine brain) ${ }^{f}$ & ++ & - & Anionic lysophospholipid, incompatible with IEF \\
\hline LPE (egg, mixed chain)g & - & - & Zwitterionic lysophospholipid; low solubility in high urea buffer \\
\hline LPG (egg, mixed chain $)^{h}$ & ++ & - & Anionic lysophospholipid, incompatible with IEF \\
\hline LPA (egg, mixed chain) ${ }^{i}$ & ++ & - & Anionic lysophospholipid, incompatible with IEF \\
\hline cardiolipin (bovine heart) & ++ & - & Anionic lipid, incompatible with IEF, low solubility in high urea buffer \\
\hline 5,7-docosadiynoic acid & - & - & Synthetic fatty acid; low solubility in high urea buffer \\
\hline lauric acid & +++ & ++ & Medium chain fatty acid; low solubility in high urea buffer \\
\hline free fatty acids (mixed) & - & - & Mixed natural fatty acids; low solubility in high urea buffer \\
\hline DODAPi & +++ & - & Cationic lipid used as a transfection reagent [37], IEF incompatible \\
\hline I-oleoyl-sn-glycerol & + & - & Uncharged monoacylated lipid, very low solubility in high urea buffer \\
\hline $\mathrm{C}_{12} \mathrm{E}_{8} \mathrm{k}$ & + & - & Nonionic detergent used to study membrane proteins [38] \\
\hline DL- $\alpha$-O-benzylglycerol & + & - & Amphipathic cyclic glycerol conjugate \\
\hline tryptophol & ++ & - & Amphipathic heterocyclic metabolite of tryptophan; ionizable at low $\mathrm{pH}$ \\
\hline
\end{tabular}

\footnotetext{
a octanoyl-N-methylglucamide

b nonanoyl-N-methylglucamide

c decanoyl-N-methylglucamide

d N-decyl-N,N-dimethyl-3-ammonio-I-propanesulfonate

e L- $\alpha$-lysophosphatidylcholine

f $\mathrm{L}-\alpha-$-lysophosphatiylserine

$\mathrm{g} \mathrm{L}-\alpha$-lysophosphatidylethanolamine

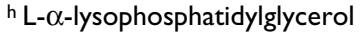

i L- $\alpha$-lysophosphatidic acid

i I,2-dioleoyloxy-3-(dimethylamino)propane

$k$ octaethylene glycol monododecyl ether
}

RBC membranes extracted with the $4 \%$ total detergent mixtures of CHAPS and LPC showed general evidence of improved spot densities (Fig. 2B), as well as specific improvements in terms of reduced horizontal streaking in the intermediate molecular weight region (Fig. 2A). Automated spot detection and quantitative comparative analysis using Progenesis Workstation software identified specific changes in the protein pattern. Specific areas of the gels showed improvement relative to parallel $4 \%$ CHAPS control gels (Fig. 2Bi-iv). In particular, a prominent spot corresponding to band III was clearly observed $[15,16]$, as well as a $2.2 \pm 0.1$ - fold increase in the density of a string of spots relative to the CHAPS extract (Fig. 2Bii). Three additional unique spots were observed when extracted with 3\% CHAPS : 1\% LPC (Fig 2Biii-iv). RBC membrane samples extracted with 3\% CHAPS : $1 \%$ MEGA 10 (Fig. 2C) yielded protein maps of generally equivalent resolution to both the 3\% CHAPS : 1\% LPC and the 4\% CHAPS maps, but did not resolve the protein band III as effectively as 3\% CHAPS : $1 \%$ LPC.

In order to examine the overlapping effects of both these test detergents, but ameliorate the observed losses of protein, $0.5 \%$ of each was mixed with 3\% CHAPS and tested in the extraction and 2-DE analysis of RBC membrane proteins (Fig. 2D). Extraction with 3\% CHAPS : 0.5\% LPC : 0.5\% MEGA 10 does not yield the extent of differences identified in the 3\% CHAPS : $1 \%$ LPC extracted condition, although the maps show general improvements over the control CHAPS condition that correlate with the improvements seen in the two individual detergent extractions (Figs. 2B, C). The density of the indicated string of proteins was increased an average of $1.7 \pm 0.2$ - fold over CHAPS (Fig. 2Dii). In general then, the addition of LPC to 


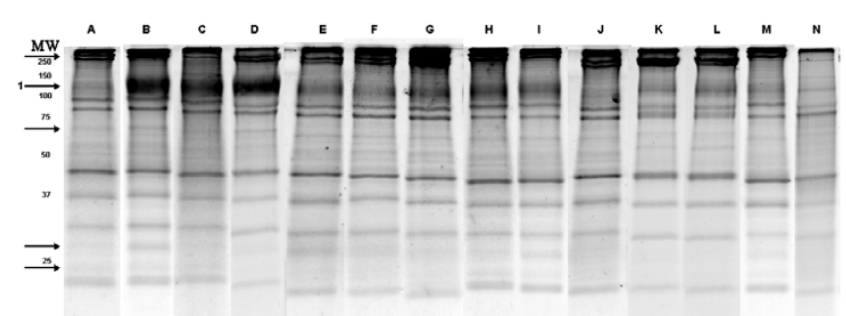

Figure I

Composite of ID SDS-PAGE analyses of RBC ghost membranes extracted with A) $4 \%$ CHAPS, B) $2 \%$ SDS, C) $2 \%$ LPC, D) $2 \%$ lauric acid, E) 2\% trans, trans-farnesol, F) 2\% MEGA 8 , G) $2 \%$ MEGA 9, H) 2\% MEGA I0, I) 2\% I,2 dioleoyloxy -3(dimethylamino)propane, J) 2\% SB 3-I0 (Sigma), K) 2\% SB 3I0 (Calbiochem), L) CI2E8, M) I-oleoyl-sn-glycerol, N) DL$\alpha$-O-benzylglycerol. Arrows indicate notable differences between extractions including I, the multiple transmembrane spanning protein band III.

the extraction buffer enhances both protein recovery and resolution in the subsequent $2 \mathrm{D}$ protein maps.

Our initial findings using the RBC membrane as a model system lead us to expand the analyses to additional tissue types. Mouse brain membranes [27] and mouse liver membranes were chosen due to their availability, and broad international interest in improved analyses of these tissue proteomes.

\section{2-DE analysis of mouse brain membrane}

Adult mouse brain membrane samples were subjected to the final four extraction conditions (Fig. 3) in order to further test the results obtained in RBC membranes (Fig. 2). Overall the results were quite similar to those obtained in the tests on RBC membranes. Extraction of mouse brain membranes with 3\% CHAPS : 1\% LPC (Fig. 3B) showed improvement of spot number, density and resolution compared to extraction with 4\% CHAPS alone (Fig. 3A); quantitative analysis indicated specific areas of significant improvement (Fig. 4). Automated analysis identified $13 \pm$ 3 novel spots that were reproducibly detected primarily in the low molecular weight and basic extreme regions of the gel (Fig. 4B; blue arrows indicate novel spots). Additionally, 5 spots were identified that significantly increased in volume an average $7.0 \pm 3.4$-fold, and increased in density $2.8 \pm 0.9$-fold compared to the $4 \%$ CHAPS condition (Fig. 4B; green arrows indicate increased recovery). Of the $15 \pm 2$ novel spots detected in the 3\% CHAPS : $1 \%$ MEGA 10 condition (Fig. 4C), most were also observed in the 3\% CHAPS : $1 \%$ LPC condition. Overall, of the same 5 spots showing increased recovery, the volume increased $5.8 \pm$ 2.5-fold, while density increased $3.2 \pm 0.9$-fold (Fig 4C; green arrows). Extraction of mouse brain membrane with
3\% CHAPS : 0.5\% LPC : 0.5\% MEGA 10 (Fig. 3D) showed an additive effect on spot number. Spots recovered in both 3\% CHAPS : 1\% LPC and 3\% CHAPS : 1\% MEGA 10 were also detected in the combined extraction system. 13 \pm 1 novel spots were detected relative to control, and the 5 previously identified spots increased in volume $6.4 \pm$ 0.4 -fold and density was increased $2.6 \pm 0.6$-fold (Fig 4D; green arrows). The nature of the recovery of these protein spots in 3\% CHAPS : 0.5\% LPC : 0.5\% MEGA 10 reveals the specific action of the two detergents - LPC and MEGA 10 working in concert. Only one selective loss of a protein spot was observed in relation to this recovery of unique spots (Fig. 4Ai); this loss is the result of a specific action shared by the two alternate detergents as opposed to a result of the difference in CHAPS concentration during extraction since this protein was not recovered even after extraction with 5\% total detergent (4\% CHAPS : $0.5 \%$ LPC : $0.5 \%$ MEGA 10) (data not shown). This loss implies some specific action of the alternate detergents that prevent the extraction of this particular protein, or possibly an alteration in the electrophoretic mobility of this protein in the first dimension by means of increasing or decreasing the number of exposed ionizable residues. Together, the results of the RBC membrane and mouse brain membrane extractions show that simple combinations of zwitterionic detergents (CHAPS and MEGA 10) with a zwitterionic lipid (LPC) are generally more effective at extracting membrane proteins and maintaining protein solubility during first dimension IEF than are standard CHAPS-based extraction conditions.

\section{Additional 2-DE Analyses}

Interestingly, extracting mouse liver membranes with the same detergent combinations described above resulted in protein maps that were highly similar, with very limited improvements. Automated analysis indicated almost complete overlap of the resulting 2-DE protein patterns (Fig 5), with the specific and substantial recovery of one additional protein spot. We interpret the marked similarity in these liver protein profiles, relative to the differences seen in the RBC and brain samples, to be due to variability between tissues in terms of relative homogenization/ extraction efficiency and compatibility with our current buffer system.

To control for possible differences arising from the changing CHAPS concentration in these test extraction buffers, mouse brain membranes were also extracted with 5\% total detergent (5\% CHAPS or $4 \%$ CHAPS : $0.5 \%$ LPC : $0.5 \%$ MEGA 10 ) and analyzed in parallel with membranes extracted with $4 \%$ total detergent. No significant difference in overall spot pattern or specific differences as described above was observed between the 5\% and the $4 \%$ total detergent mixtures (data not shown), indicating that the differences described here are specifically 

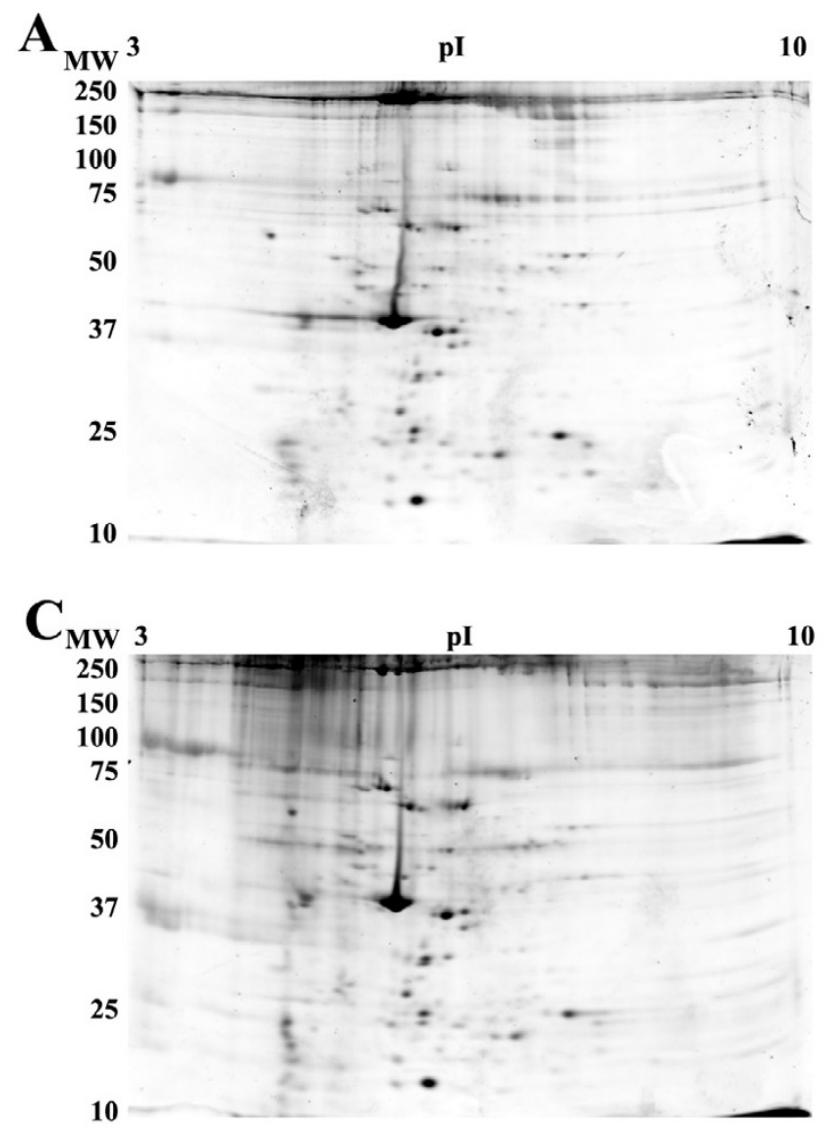
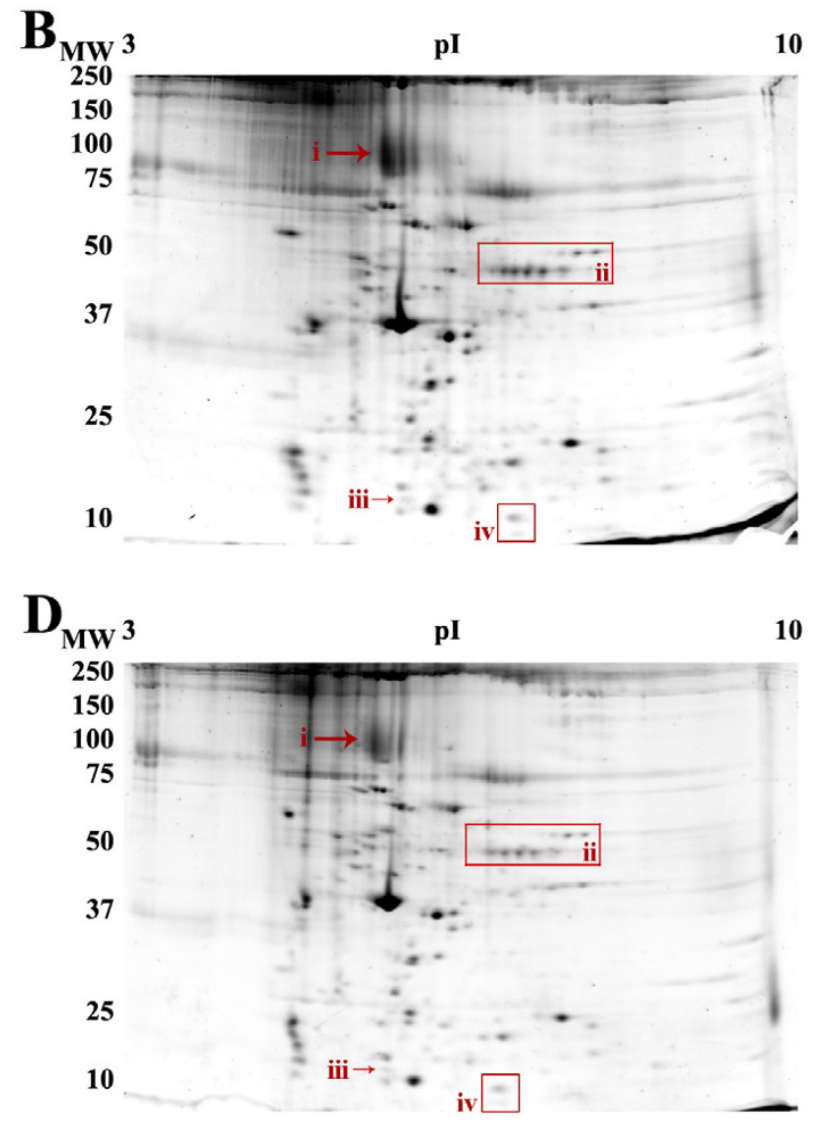

Figure 2

2-DE of RBC ghost membranes extracted with A) 4\% CHAPS, B) 3\% CHAPS : I\% LPC, C) 3\% CHAPS : I\% MEGA I0, D) 3\% CHAPS : $0.5 \%$ LPC : $0.5 \%$ MEGA 10 . Extractions were carried out in buffer with $8 \mathrm{M}$ urea, $2 \mathrm{M}$ thiourea, protease inhibitor cocktail, and the indicated detergent for I hour on ice. Gels are representative of three independent experiments. Roman numerals indicate areas of improvement including i, the multiple transmembrane spanning protein band III.

attributable to the addition of LPC and MEGA 10 as solubilizing agents. Indeed, overall, membrane protein patterns were generally of somewhat lower resolution when the CHAPS concentration or total detergent concentration was increased to $5 \%$.

\section{Protein Quantification}

During initial experiments we found total protein load to be the most significant variable confounding quantitative analyses. As such, great care was taken to ensure that the analyses meaningfully tested protein extraction and solubilization efficiency, in isolation from complicating variables. Simply, the goal was to compare reagents and conditions, not to compare different final total protein loads by 2-DE. Initially many protein samples were quantified using a modified Folin total protein assay (RC DC Protein Assay kit, BioRad). Colourimetric assays of this type (eg. Bradford, Lowry, BCA, and so forth) perform acceptably under many circumstances requiring routine normalization of a series of very similar samples. However one of several limitations of such total protein assays is a marked sensitivity to interfering substances, including components of typical IEF solubilization solutions such as detergents, reducing agents, and urea. In our experiments, detergents and detergent concentrations were systematically altered and combined. Not unexpectedly, we observed substantial variability in the results of the total protein assay, depending upon the solubilizing reagents present. The complications of applying systematic corrective controls, or of preparing separate standard curves for each of the solubilization conditions tested, simply increased the potential for error. Regardless, separate standard curves are not even feasible in the case of the RC DC assay, as urea causes a saturating false positive signal. 


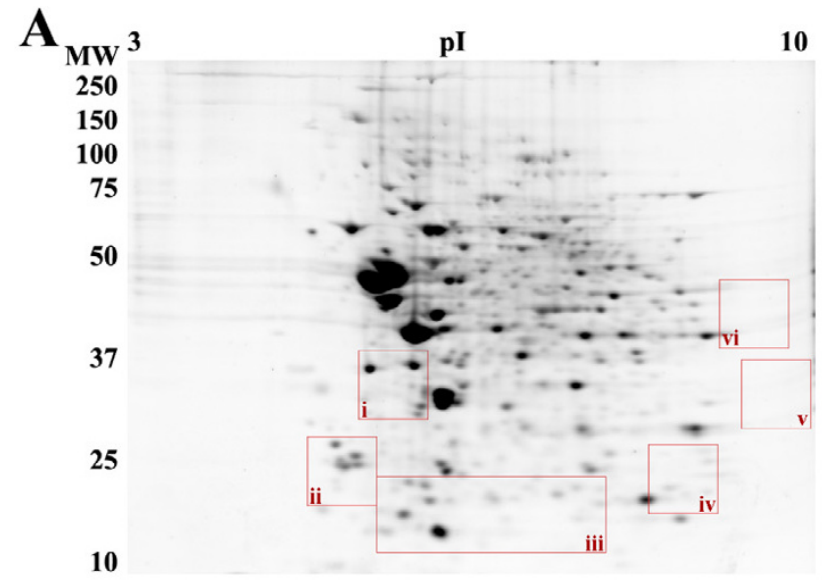

0
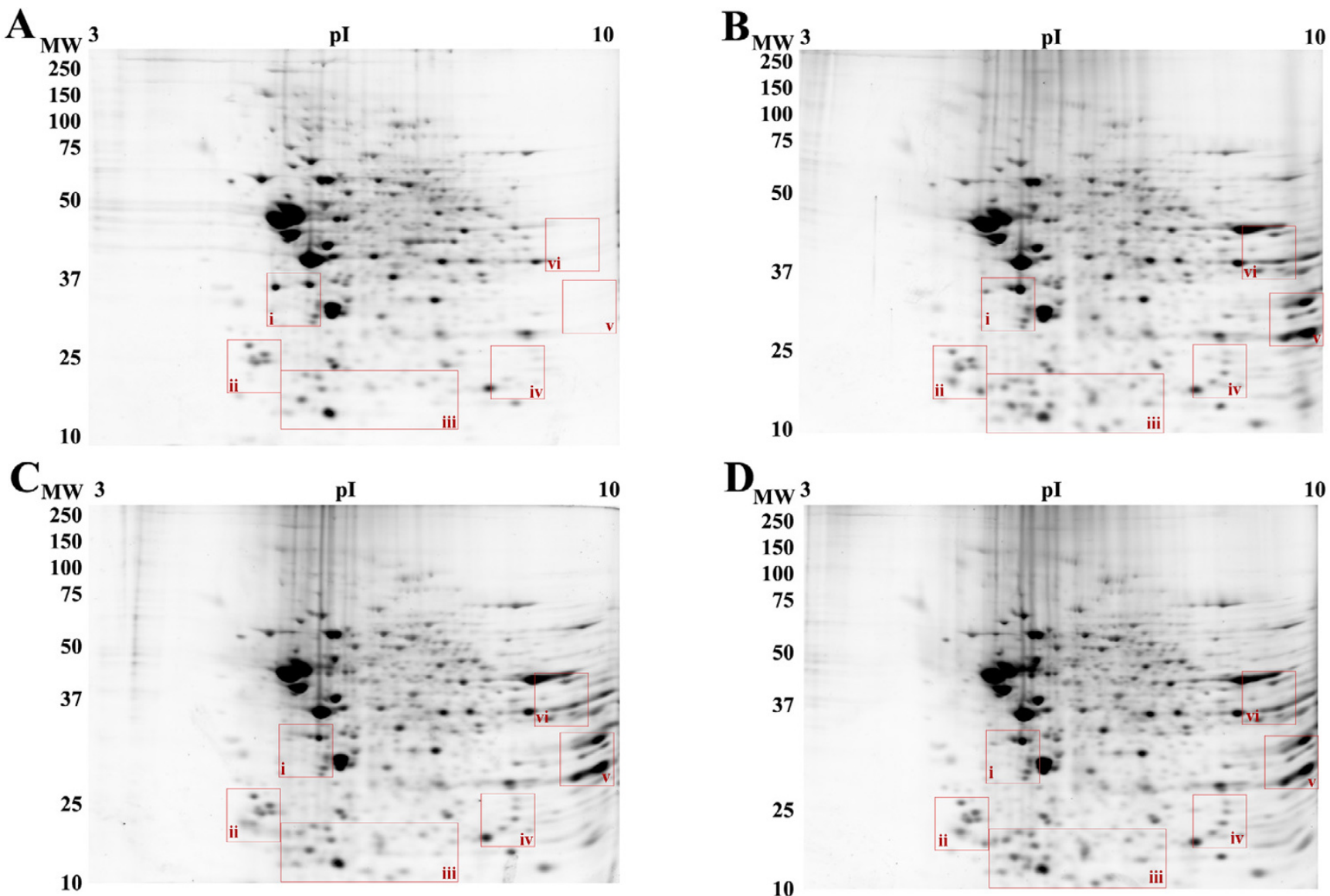

\section{Figure 3}

2-DE of mouse brain membranes extracted with A) 4\% CHAPS, B) 3\% CHAPS : I\% LPC, C) $3 \%$ CHAPS : I\% MEGA I0, D) $3 \%$ CHAPS : $0.5 \%$ LPC : $0.5 \%$ MEGA 10. Extractions were carried out as for Fig. 2. Gels are representative of three independent experiments. Areas defined with Roman numerals are shown in Fig. 4.

We have found that the EZQ Protein Quantitation kit (Molecular Probes) is insensitive to the nature and concentrations of detergent in all samples tested. In this assay format, the immobilized protein sample is washed exhaustively with methanol to remove components of the solubilization solution prior to addition of the fluorescent protein detection reagent. Thus, the chemistry of the assay proceeds in the absence of potentially confounding contaminants. In extensive comparisons, there were no significant differences in standard protein assay curves regardless of the type or quantity of detergent included (data not shown). Additionally, the method proved quite sensitive (routine detection of $0.030 \mu \mathrm{g}$ of total protein/ spot, or $15 \mu \mathrm{g} / \mathrm{ml}$ ); this is fully 10 -fold more sensitive and requires 4 -fold less material than the RC DC Assay. Thus, as the chemistry of the assay was not altered under our different experimental conditions, we are confident that the improvements observed in our final protein maps were truly the result of differences in extraction and solubilization efficiency, and not artifacts generated by erroneous total protein assays leading to inconsistent total protein IEF loads between different test conditions. Although the EZQ protein assay certainly has its caveats, not least of which is cost, it does offer distinct benefits that support its utility in these and other ongoing proteomic analyses.

\section{Conclusion}

In order to optimize recovery of hydrophobic proteins for 2-DE, we have sought a simple, direct solution to the problem of protein extraction and solubility during IEF. The systematic screening and combination of commercially available detergents offers a direct, inexpensive, and convenient method for optimizing the conditions of IEF without entering into the complexities of a systematic 
A

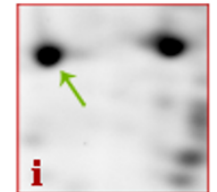

B

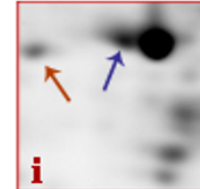

C

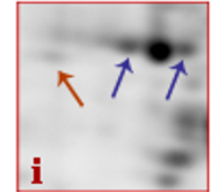

D

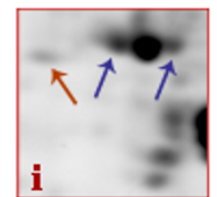

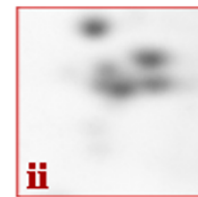
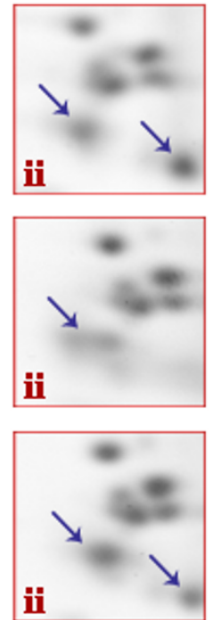
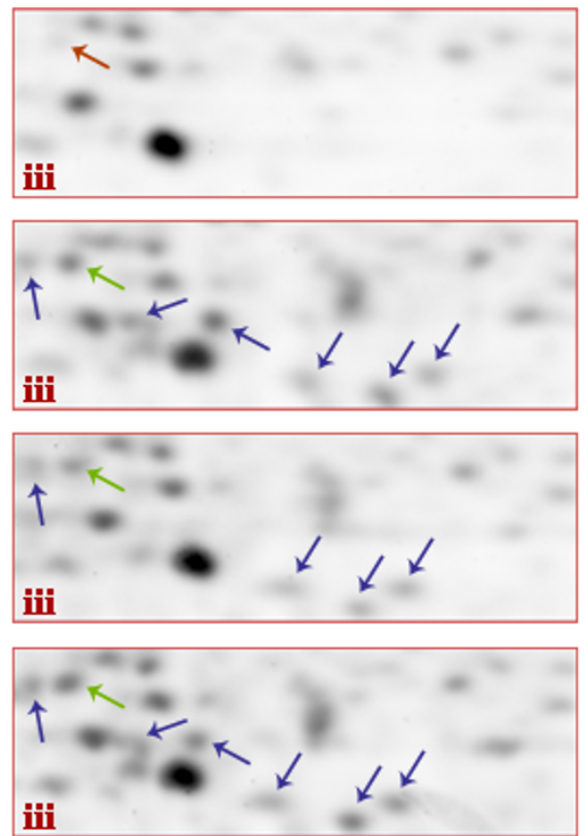
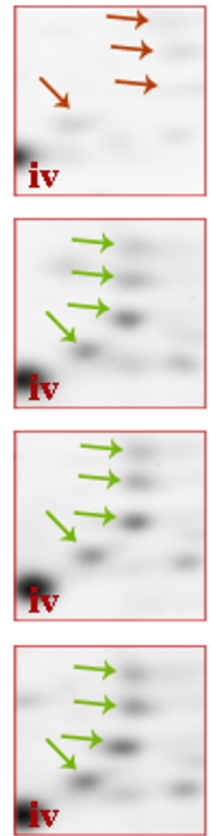
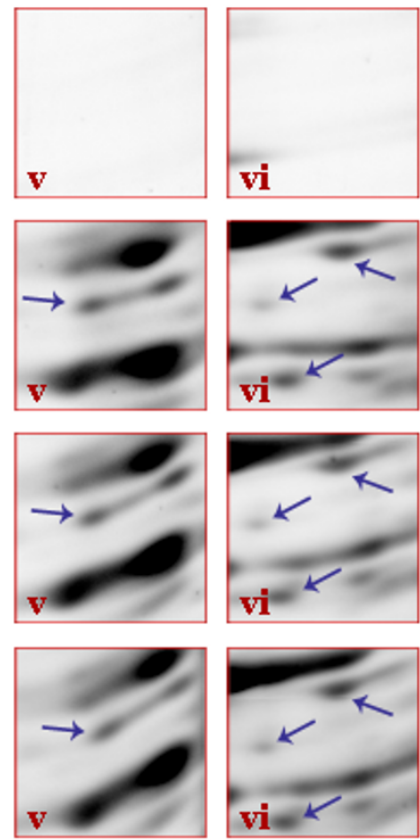

Figure 4

Enlargement and contrast of selected regions after 2-DE of mouse brain membranes (see areas defined in Fig 3). Areas i-vi show selective increases in spot number, resolution, and density. Samples were extracted with A) $4 \%$ CHAPS, B) $3 \%$ CHAPS : I\% LPC, C) 3\% CHAPS : I\% MEGA 10, D) 3\% CHAPS : 0.5\% LPC : $0.5 \%$ MEGA 10. Results are representative of three independent experiments. Green arrows indicate spots showing increased volume and density, red arrows indicate decrease, blue arrows indicate novel spots.
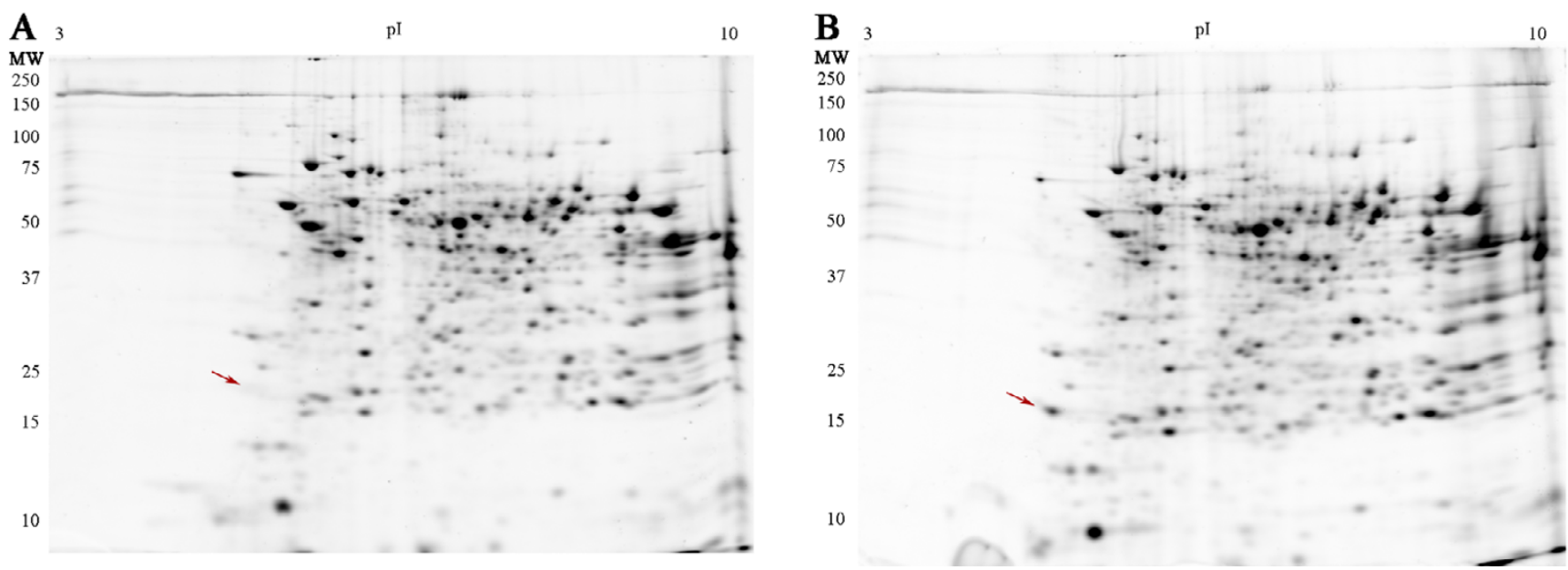

Figure 5

2-DE gels of mouse liver membranes extracted with A) 4\% CHAPS, B) 3\% CHAPS : I\% LPC. Extractions were carried out as for Fig. 2. Gels are representative of three independent experiments. Arrow indicates specific differences between gels. 
synthesis of new detergents based on specific base molecules, or the potential losses or modification of proteins associated with solvent extraction techniques. Coupled with our ability to effectively analyze membrane proteomes using 2-DE [27] the resulting findings should also prove of use in defining optimized combinations of extraction reagents for use with alternate protein separation protocols.

Based on the hypothesis that highly lipophilic molecules (albeit at lower total concentrations than can be achieved with the more standard detergents), might better mimic native lipid-membrane protein interactions and thus improve protein solubilization, we found that LPC can substantially augment the extraction of membrane proteins from different sources. This finding does not obviate the need for optimization of extraction and 2-DE conditions for different samples, but does provide a powerful, widely available and reasonably priced alternative that can be readily tested in parallel with more routine solubilization reagents. Rigorous testing of protein assays ensured that these findings reflect a true effect on extraction and protein solubility, rather than an artifact of inconsistent protein loads between different 2-DE analyses. Notably, LPC and MEGA 10 provided particularly marked improvements in the resolution of the mouse brain membrane proteome.

\section{Methods \\ Reagents}

L- $\alpha$-lysophosphatidylcholine lauroyl, urea, tris acetate, lauric acid, pH 3-10 ampholytes, ammonium persulfate, decyl-N,N-dimethyl-3-ammonio-1-propanesulfonate (SB 3-10), amidosulfobetaine-14 (ASB-14), DL- $\alpha$-O-benzylglycerol, tributylphosphine (TBP), HEPES, sodium orthovanadate, staurosporine, cantharidin, and components of the broad spectrum protease inhibitor cocktail [28] were purchased from Sigma (St. Louis, Missouri). IPG strips (pH 3-10), 30\% acrylamide/bisacrylamide solution, low melting agarose, Sypro Ruby, 10×TGS running buffer, RC DC Protein Assay Kit, bovine $\gamma$-globulin, and SDS were from BioRad (Hercules, California). EZQ Protein Quantitation Kit was from Molecular Probes (Eugene, OR), Zwittergent ${ }^{\circledR}$ 3-10 was from Calbiochem (La Jolla, California), and CHAPS was from Anatrace (Maumee, Ohio). 1,2-dioleoyloxy-3-(dimethylamino)propane, 5,7-docosadiynoic acid, and 1-oleoyl-snglycerol were from Toronto Research Chemicals (Toronto, Ontario). Bovine brain L- $\alpha$-lysophosphatidylserine, egg L$\alpha$-lysophosphatidylcholine, egg L- $\alpha$-lysophosphatidylethanolamine, egg L- $\alpha$-lysophosphatidylglycerol, egg L- $\alpha$-lysophosphatidic acid, bovine heart cardiolipin, and free fatty acids were from Doosan Serdary Research (Toronto, Ontario). Thiourea was from Fisher Scientific (Hampton, New Hampshire), and PBS, DTT, octanoyl-N-methylglu- camide (MEGA 8), nonanoyl-N-methylglucamide (MEGA 9), decanoyl-N-methylglucamide (MEGA 10), TEMED, glycerol, $40 \%$ acrylamide solution, and octaethylene glycol monododecyl ether $\left(\mathrm{C}_{12} \mathrm{E}_{8}\right)$ were from Bio Basic Inc. (Markham, Ontario). Narrow range ampholytes ( $\mathrm{pH} 2.5-$ 4, 3.5-5, 5-7, 7-9, and 8-9.5) were from Fluka (Buchs, Switzerland), and tryptophol and trans, trans-farnesol was from Aldrich (St. Louis, Missouri). All other chemicals were of at least analytical grade.

\section{Red Blood Cell membrane preparation}

Packed RBC were obtained from Canadian Blood Services, (Calgary, AB) and washed $3 \times$ with isotonic buffer $(20 \mathrm{mM}$ sodium phosphate $\mathrm{pH} 7.4,0.9 \% \mathrm{NaCl}$ ). $\mathrm{RBC}$ ghosts were prepared according to the method of Chernomordik [29] with slight modifications. Cells were lysed osmotically in hypotonic lysis buffer ( $5 \mathrm{mM}$ sodium phosphate $\mathrm{pH}$ 7.4, protease inhibitor cocktail [28], $5 \mathrm{mM}$ DTT) for $20 \mathrm{~min}$ utes on ice. The lysate was flash frozen in a dry ice / ethanol bath, thawed, and membranes were collected by centrifugation $\left(3000 \times \mathrm{g}, 20 \mathrm{~min}, 4^{\circ} \mathrm{C}\right)$. Pellets were washed with wash buffer $(20 \mathrm{mM}$ sodium phosphate $\mathrm{pH}$ 8.5 , protease inhibitor cocktail, $5 \mathrm{mM}$ DTT) until supernatants were clear, and then subjected to a second round of hypotonic lysis and freeze-thaw. After washing until supernatants were clear, membranes were collected by centrifugation $\left(3000 \times \mathrm{g}, 40 \mathrm{~min}, 4^{\circ} \mathrm{C}\right)$, suspended in a minimal volume of wash buffer, and stored at $-80^{\circ} \mathrm{C}$. Before extraction, membrane isolates were washed with PBS containing protease inhibitors (PBS-PI) and pelleted (3 hours, $120000 \times \mathrm{g}, 4^{\circ} \mathrm{C}$ ).

\section{Membrane preparations from mammalian tissues}

Membranes were isolated as previously described [27]. Briefly, mouse brains or livers were flash frozen after dissection and stored at $-80^{\circ} \mathrm{C}$ until needed. For the isolation of all cellular membranes, we applied a simple physical separation / fractionation protocol. Briefly, frozen tissues were thawed in hypotonic lysis buffer (20 mM HEPES pH 7.4, protease inhibitor cocktail, $10 \mathrm{mM}$ sodium orthovanadate, $4 \mu \mathrm{M}$ staurosporin, $4 \mu \mathrm{M}$ cantharidin) and manually homogenized on ice with a polyethylene pestle in a $1.5 \mathrm{~mL}$ microcentrifuge tube. The homogenate was subjected to one round of freeze-thaw $\left(-80^{\circ} \mathrm{C}\right)$, before being combined with an equal volume of $2 \times \mathrm{PBS}$ to restore isotonicity. Membranes were collected by ultracentrifugation ( 3 hours, $120000 \times \mathrm{g}, 4^{\circ} \mathrm{C}$ ), and were washed twice; pellets were resuspended in PBS-PI for each wash and collected by ultracentrifugation, as described above.

\section{Detergent Extractions}

Detergent extraction buffers were prepared for 1D (7 M urea, $2 \mathrm{M}$ thiourea, $9 \mathrm{mM}$ Tris acetate $\mathrm{pH}$ 7.0, protease inhibitor cocktail, and detergent as indicated) or 2-DE (IEF buffer 1 containing $8 \mathrm{M}$ urea, $2 \mathrm{M}$ thiourea, protease 
inhibitor cocktail, and detergent as indicated [27]). Membrane pellets were resuspended by pipetting and vortexing. Extractions were incubated for 1 hour on ice, with periodic vortexing. Any insoluble material was separated by ultracentrifugation as previously described. Solubilized samples were assayed for total protein content using the EZQ Protein Quantitation Kit (Molecular Probes, Eugene, OR).

\section{Protein Quantification}

Total protein was assayed using either the EZQ Protein Quantitation Kit or the RC DC Protein Assay Kit (BioRad, Hercules, CA). The RC DC assay was carried out according to manufacturers instructions in 96-well plates and absorbance was measured using the Wallac Victor2 Multilabel HTS Counter (PerkinElmer Life Sciences, Boston, MA). EZQ Protein Quantitation was carried out essentially according to manufacturers instructions except fluorescence was recorded by imaging on the Proexpress multiwavelength fluorescent imager (PerkinElmer, Boston MA) and spot fluorescence was quantified using ImageQuant 5.2 software (Molecular Dynamics, Sunnyvale, CA).

\section{ID SDS-PAGE}

1D SDS-PAGE was performed in mini gel format using the BioRad Protean II Electrophoresis system, essentially as described [30] with minor modifications [31]. Samples were normalized to $2 \mathrm{mg} / \mathrm{ml}$ in the appropriate extraction buffer, and then diluted 1:1 (v/v) with $2 \times$ SDS sample buffer [30]. $10 \mu \mathrm{g}$ total protein was loaded per well on $12.5 \% \mathrm{~T}$ separating gels with $5 \% \mathrm{~T}$ stacking gels, buffered with $375 \mathrm{mM}$ Tris (pH 8.8) as described [31]. Gels were run at $125 \mathrm{~V}$ for $10 \mathrm{~min}$ to stack proteins, and then the voltage was reduced to $90 \mathrm{~V}$ to completion [32].

\section{2-DE}

Samples for IEF were normalized to $2 \mathrm{mg} / \mathrm{ml}$ with the appropriate IEF buffer, then combined $1: 1(\mathrm{v} / \mathrm{v})$ with an ampholyte-containing IEF buffer ( $8 \mathrm{M}$ urea, $2 \mathrm{M}$ thiourea, $1 \% \mathrm{pH} 3-10$ broad range ampholytes, $0.2 \%$ each narrow range ampholytes ( $\mathrm{pH} 2.5-4,3.5-5,5-7,7-9$, and 8-9.5) and detergent as indicated [27]), to introduce a working concentration of ampholytes to the sample.

Samples were sequentially reduced and alkylated essentially according to Herbert et al. $[33,34]$ with some minor modifications. Briefly, the sample was reduced by the addition of TBP and DTT to final concentrations of 2.3 $\mathrm{mM}$ and $45 \mathrm{mM}$ DTT, respectively, and incubated for 1 hour at $25^{\circ} \mathrm{C}$. The reduced sample was then alkylated with $230 \mathrm{mM}$ acrylamide monomer for 1 hour at $25^{\circ} \mathrm{C}$. Immediately following alkylation, the sample was loaded onto IPG strips for passive hydration at $25^{\circ} \mathrm{C}$ (12 hours). IEF was carried out at $15^{\circ} \mathrm{C}$ using the BioRad Protean IEF
Cell; voltage was ramped linearly to $4000 \mathrm{~V}$ (2 hours) and IEF was carried out at $4000 \mathrm{~V}$ (constant) for 37500 Vhours. After focusing, IPG strips were equilibrated essentially according to the manufacturer's instructions by sequential immersion in equilibration buffer $(6 \mathrm{M}$ urea, $2 \%$ SDS (w/v), $20 \%$ glycerol (w/v), and $375 \mathrm{mM}$ Tris $\mathrm{pH}$ 8.8) containing $130 \mathrm{mM}$ DTT for 10 minutes, followed by equilibration buffer with $350 \mathrm{mM}$ acrylamide monomer for 10 minutes. Following equilibration, IPG strips were loaded onto $12.5 \% \mathrm{~T}$ separating gels with $5 \% \mathrm{~T}$ stacking gels (buffered as described for 1D) and sealed in place with an agarose overlay ( $0.5 \%$ low melting agarose, $0.1 \%$ SDS and $375 \mathrm{mM}$ Tris $\mathrm{pH}$ 8.8). SDS-PAGE was otherwise carried out as described for 1D SDS-PAGE.

\section{Image analysis}

After electrophoresis, gels were fixed in $10 \%$ methanol, $7 \%$ acetic acid for 1 hour, washed thoroughly with water and stained with Sypro Ruby overnight. Gels were visualized using the Proexpress multiwavelength fluorescent imager (PerkinElmer, Boston MA). Quantitative image analysis was performed using Progenesis Workstation 2004 (Nonlinear Dynamics, Cambridge, UK). Parallel sets of gels were warped and matched by automated analysis, and volumes were normalized to a single spot consistent in size, shape, density and location across all gels.

\section{Abbreviations used}

LPC (12:0 L- $\alpha$-lysophosphatidylcholine), LPG (L- $\alpha$-lysophosphatidylglycerol), LPE (L- $\alpha$-lysophosphatidylethanolamine), LPS (L- $\alpha$-lysophosphatidylserine), MEGA 8 (octanoyl-N-methylglucamide), MEGA 9 (nonanoyl-Nmethylglucamide), MEGA 10 (decanoyl-N-methylglucamide), SB 3-10 (N-decyl-N,N-dimethyl-3-ammonio-1propanesulfonate), ASB-14 (amidosulfobetaine-14), C12E8 (octaethylene glycol monododecyl ether), RBC (red blood cell)

\section{Competing interests}

The author(s) declare that they have no competing interests.

\section{Authors' Contributions}

MAC supervised the SDS-PAGE analysis, designed and carried out the 2-DE analyses, data acquisition and interpretation and prepared the manuscript. RHB contributed to the experimental design, sample preparation, 2-DE analyses, and participated in drafting the manuscript. JCL and $\mathrm{KKH}$ designed and carried out the SDS-PAGE analysis. JRC conceived and planned the study, and assisted in interpretation of data and final preparation of the manuscript. All authors read and approved the final manuscript. 


\section{Acknowledgements}

The authors would like to thank Tiffany Rice, Tammy Wilson, and Dr. V. Wee Yong for kindly providing mouse tissues. We would also like to thank Jeff Lamb, Dr. Sina Ahmadi Pirshahid, Marlies Ernst, and all the members of the Coorssen lab for helpful discussions and advice. J.R.C. acknowledges support from the Canadian Institutes of Health Research, the Canada Foundation for Innovation, the Alberta Heritage Foundation for Medical Research, the Alberta Network for Proteomics Innovation, and the Heart and Stroke Foundation of Canada.

\section{References}

I. Perez-Reyes E, Wei XY, Castellano A, Birnbaumer L: Molecular diversity of L-type calcium channels. Evidence for alternative splicing of the transcripts of three non-allelic genes. J Biol Chem 1990, 265:20430-20436.

2. Strausberg RL, Feingold EA, Grouse LH, Derge JG, Klausner RD, Collins FS, Wagner L, Shenmen CM, Schuler GD, Altschul SF, Zeeberg B, Buetow KH, Schaefer CF, Bhat NK, Hopkins RF, Jordan H, Moore T, Max SI, Wang J, Hsieh F, Diatchenko L, Marusina K, Farmer AA, Rubin GM, Hong L, Stapleton M, Soares MB, Bonaldo MF, Casavant TL, Scheetz TE, Brownstein MJ, Usdin TB, Toshiyuki S, Carninci P, Prange C, Raha SS, Loquellano NA, Peters GJ, Abramson RD, Mullahy SJ, Bosak SA, McEwan PJ, McKernan KJ, Malek JA, Gunaratne PH, Richards S, Worley KC, Hale S, Garcia AM, Gay LJ, Hulyk SW, Villalon DK, Muzny DM, Sodergren EJ, Lu X, Gibbs RA, Fahey J, Helton E, Ketteman M, Madan A, Rodrigues S, Sanchez A, Whiting M, Madan A, Young AC, Shevchenko Y, Bouffard GG, Blakesley RW, Touchman JW, Green ED, Dickson MC, Rodriguez AC, Grimwood J, Schmutz J, Myers RM, Butterfield YS, Krzywinski MI, Skalska U, Smailus DE, Schnerch A, Schein JE, Jones SJ, Marra MA: Generation and initial analysis of more than 15,000 full-length human and mouse cDNA sequences. Proc Natl Acad Sci U S A 2002, 99: I6899-I6903.

3. Anzai T, Shiina T, Kimura N, Yanagiya K, Kohara S, Shigenari A, Yamagata T, Kulski JK, Naruse TK, Fujimori Y, Fukuzumi Y, Yamazaki M, Tashiro H, Iwamoto C, Umehara Y, Imanishi T, Meyer A, Ikeo K, Gojobori T, Bahram S, Inoko H: Comparative sequencing of human and chimpanzee MHC class I regions unveils insertions/deletions as the major path to genomic divergence. Proc Natl Acad Sci U S A 2003, I00:7708-77I3.

4. Nandakumar MP, Shen J, Raman B, Marten MR: Solubilization of trichloroacetic acid (TCA) precipitated microbial proteins via naOH for two-dimensional electrophoresis. J Proteome Res 2003, 2:89-93.

5. Mastro R, Hall M: Protein delipidation and precipitation by trin-butylphosphate, acetone, and methanol treatment for isoelectric focusing and two-dimensional gel electrophoresis. Anal Biochem 1999, 273:313-315.

6. Aivaliotis M, Corvey C, Tsirogianni I, Karas M, Tsiotis G: Membrane proteome analysis of the green-sulfur bacterium Chlorobium tepidum. Electrophoresis 2004, 25:3468-3474.

7. Hagopian K: Preparative electrophoretic method for the purification of a hydrophobic membrane protein: subunit $c$ of the mitochondrial ATP synthase from rat liver. Anal Biochem 1999, 273:240-25I.

8. Ruppert C, Kavermann H, Wimmers S, Schmid R, Kellermann J, Lottspeich $\mathrm{F}$, Huber $\mathrm{H}$, Stetter KO, Muller V: The proteolipid of the A(I)A(0) ATP synthase from Methanococcus jannaschii has six predicted transmembrane helices but only two protontranslocating carboxyl groups. J Biol Chem 1999, 274:2528I-25284.

9. Skalidis G, Trifilieff E, Luu B: Selective extraction of the DM-20 brain proteolipid. I Neurochem 1986, 46:297-299.

10. Zuo X, Speicher DW: A method for global analysis of complex proteomes using sample prefractionation by solution isoelectrofocusing prior to two-dimensional electrophoresis. Anal Biochem 2000, 284:266-278.

II. Vuong GL, Weiss SM, Kammer W, Priemer M, Vingron M, Nordheim $A$, Cahill MA: Improved sensitivity proteomics by postharvest alkylation and radioactive labelling of proteins. Electrophoresis 2000, 21 :2594-2605.

12. Wallin E, von HG: Genome-wide analysis of integral membrane proteins from eubacterial, archaean, and eukaryotic organisms. Protein Sci 1998, 7:1029-1038.
13. Rabilloud T, Blisnick T, Heller M, Luche S, Aebersold R, Lunardi J, Braun-Breton C: Analysis of membrane proteins by twodimensional electrophoresis: comparison of the proteins extracted from normal or Plasmodium falciparum-infected erythrocyte ghosts. Electrophoresis 1999, 20:3603-3610.

14. Santoni V, Kieffer S, Desclaux D, Masson F, Rabilloud T: Membrane proteomics: use of additive main effects with multiplicative interaction model to classify plasma membrane proteins according to their solubility and electrophoretic properties. Electrophoresis 2000, 21:3329-3344.

15. Luche S, Santoni V, Rabilloud T: Evaluation of nonionic and zwitterionic detergents as membrane protein solubilizers in twodimensional electrophoresis. Proteomics 2003, 3:249-253.

16. Tastet C, Charmont S, Chevallet M, Luche S, Rabilloud T: Structureefficiency relationships of zwitterionic detergents as protein solubilizers in two-dimensional electrophoresis. Proteomics 2003, 3: III-121.

17. Kern R, Joseleau-Petit D, Chattopadhyay MK, Richarme G: Chaperone-like properties of lysophospholipids. Biochem Biophys Res Commun 200I, 289: I 268-1274.

18. Nekrasova E, Sosinskaya A, Natochin M, Lancet D, Gat U: Overexpression, solubilization and purification of rat and human olfactory receptors. Eur J Biochem 1996, 238:28-37.

19. Perez-Gil J, Cruz A, Casals C: Solubility of hydrophobic surfactant proteins in organic solvent/water mixtures. Structural studies on SP-B and SP-C in aqueous organic solvents and lipids. Biochim Biophys Acta 1993, I I 68:26I-270.

20. Navarre C, Degand H, Bennett KL, Crawford JS, Mortz E, Boutry M: Subproteomics: identification of plasma membrane proteins from the yeast Saccharomyces cerevisiae. Proteomics 2002, 2: $1706-17 \mid 4$.

21. Santoni V, Molloy M, Rabilloud T: Membrane proteins and proteomics: un amour impossible? Electrophoresis 2000, 2 I: 1054-1070.

22. Witzmann F, Jarnot B, Parker D: Dodecyl maltoside detergent improves resolution of hepatic membrane proteins in twodimensional gels. Electrophoresis 199|, I 2:687-688.

23. Lux SE, John KM, Kopito RR, Lodish HF: Cloning and characterization of band 3 , the human erythrocyte anion-exchange protein (AEI). Proc Natl Acad Sci U S A 1989, 86:9089-9093.

24. Rabilloud T, Gianazza E, Catto N, Righetti PG: Amidosulfobetaines, a family of detergents with improved solubilization properties: application for isoelectric focusing under denaturing conditions. Anal Biochem 1990, I85:94-102.

25. Navarrete R, Serrano R: Solubilization of yeast plasma membranes and mitochondria by different types of non-denaturing detergents. Biochim Biophys Acta 1983, 728:403-408.

26. Chevallet M, Santoni V, Poinas A, Rouquie D, Fuchs A, Kieffer S, Rossignol M, Lunardi J, Garin J, Rabilloud T: New zwitterionic detergents improve the analysis of membrane proteins by twodimensional electrophoresis. Electrophoresis 1998 19:1901-1909.

27. Butt RH, Coorssen JR: Postfractionation for enhanced proteomic analyses: Routine electrophoretic methods increase the resolution of standard 2D-PAGE. J Proteome Res 2005, 4(3982-91 [http://dx.doi.org//0.1021/pr050054d]

28. Coorssen JR, Blank PS, Tahara M, Zimmerberg J: Biochemical and functional studies of cortical vesicle fusion: the SNARE complex and Ca2+ sensitivity. J Cell Biol 1998, I 43: | 845- I857.

29. Chernomordik LV, Sowers AE: Evidence that the spectrin network and a nonosmotic force control the fusion product morphology in electrofused erythrocyte ghosts. Biophys J 1991, 60:1026-1037.

30. Laemmli UK: Cleavage of structural proteins during the assembly of the head of bacteriophage T4. Nature I970, 227:680-685.

31. Cannon-Carlson S, Tang J: Modification of the Laemmli sodium dodecyl sulfate-polyacrylamide gel electrophoresis procedure to eliminate artifacts on reducing and nonreducing gels. Anal Biochem 1997, 246: |46-|48.

32. Coorssen JR, Blank PS, Albertorio F, Bezrukov L, Kolosova I, Backlund PS ], Zimmerberg ]: Quantitative femto- to attomole immunodetection of regulated secretory vesicle proteins critical to exocytosis. Anal Biochem 2002, 307:54-62.

33. Herbert B, Galvani M, Hamdan M, Olivieri E, MacCarthy J, Pedersen $S$, Righetti PG: Reduction and alkylation of proteins in 
preparation of two-dimensional map analysis: why, when, and how? Electrophoresis 200I, 22:2046-2057.

34. Herbert BR, Molloy MP, Gooley AA, Walsh BJ, Bryson WG, Williams $\mathrm{KL}$ : Improved protein solubility in two-dimensional electrophoresis using tributyl phosphine as reducing agent. Electrophoresis 1998, 19:845-85I.

35. Lundahl P, Greijer E, Lindblom H, Fagerstam LG: Fractionation of human red cell membrane proteins by ion-exchange chromatography in detergent on Mono $Q$, with special reference to the glucose transporter. J Chromatogr 1984, 297: I 29-I37.

36. Poole RC, Halestrap AP: Reconstitution of the L-lactate carrier from rat and rabbit erythrocyte plasma membranes. Biochem J 1988, 254:385-390.

37. Semple SC, Klimuk SK, Harasym TO, Dos SN, Ansell SM, Wong KF, Maurer N, Stark H, Cullis PR, Hope MJ, Scherrer P: Efficient encapsulation of antisense oligonucleotides in lipid vesicles using ionizable aminolipids: formation of novel small multilamellar vesicle structures. Biochim Biophys Acta 200I, 15 I 0:152-166.

38. Martin DW: Active unit of solubilized sarcoplasmic reticulum calcium adenosinetriphosphatase: an active enzyme centrifugation analysis. Biochemistry 1983, 22:2276-2282.

Publish with Bio Med Central and every scientist can read your work free of charge

"BioMed Central will be the most significant development for disseminating the results of biomedical research in our lifetime. "

Sir Paul Nurse, Cancer Research UK

Your research papers will be:

- available free of charge to the entire biomedical community

- peer reviewed and published immediately upon acceptance

- cited in PubMed and archived on PubMed Central

- yours - you keep the copyright 